\title{
A unified approach to closed-loop plant identification and direct controller reduction
}

\author{
I.D. Landau ${ }^{(1)}$, A. Karimi ${ }^{(2)}$ \\ (1) Laboratoire d'Automatique de Grenoble (CNRS-INPG-UJF), ENSIEG, BP. 46 \\ 38402 St. Martin d'Hères, FRANCE; e-mail: landau@lag.ensieg.inpg.fr \\ (2) Electrical Engineering Department of Sharif University of Technology, Azadi Ave., \\ 11365-9363 Teheran, IRAN ; e-mail : karimi@sina.sharif.ac.ir
}

\begin{abstract}
Closed loop output error identification algorithms and recently developed algorithm for direct closed loop estimation of reduced order controllers [3, 5] despite their diversity have in fact a unifying basic ground which will be enhanced.

The paper also will explore the interaction between closed loop plant model identification and direct estimation in closed loop of reduced order controllers. The following problem will be addressed: what closed loop plant model identification should be used when a criterion for direct controller reduction is given?
\end{abstract}

Keywords: system identification, closed loop identification, controller reduction.

\section{Introduction}

Closed loop output error (CLOE) identification algorithms $[4,6]$ allow to identify an approximate design model which features a high accuracy in the critical regions for control design. Effectively the frequency distribution of the asymptotic error (bias) between the estimated and the true model is heavily weighted by the magnitude of the sensitivity function of the true system which explains why this desired property is obtained.

In fact the algorithms search for a plant model which minimizes a 2-norm error between the true closed loop transfer function and the simulated closed loop transfer function containing the final estimated model.

Asymptotic frequency bias error distribution can be obtained indicating clearly that the bias is small where some sensitivity functions are high and in addition this bias error is not affected by the measurement noise $[4,1]$.

Several configurations for the closed loop output output error identification can be established. While the parameter adaptation algorithms (PAA) is basically the same, the identification criterion and the asymptotic properties of the estimated model will be different.
It has been shown in the papers $[5,3]$ that direct controller reduction can be performed by identification in closed loop either using simulated data or real data (which is a unique feature of this approach with respect to other approaches to direct controller reduction). To proceed one need to know the nominal controller and to have an estimated plant model (either the model used for design or a model identified on site in open or closed loop). The algorithms developed for the estimation of reduced order controllers lead to reduced order controllers which minimize a 2 -norm of the difference between the nominal sensitivity function and the one obtained with the reduced order controller with the property that the error between the reduced order controller and the nominal controller is small at the frequencies where some sensitivity functions are high.

Several configurations can be considered each corresponding to specific controller reduction criterion. However the P.A.A. is in fact basically the same. In addition, they are similar to those used for closed loop plant model identification and they can be obtained by a direct substitution i.e.:

estimated plant model $\longrightarrow$ estimated controller; nominal controller $\longrightarrow$ plant model.

The only differences will occur at the effective implementation of the algorithms since the controller has a direct transfer term while the plant model has at least one step delay.

The first objective of the paper is to survey the various possible configuration for closed loop plant model identification and estimation of reduced order controllers and to show what are the basic algorithms.

The second objective of the paper is to explore the interaction between closed loop plant model identification and direct estimation in closed loop of reduced order controllers. The following problem will be addressed: what closed loop plant model identification should be used when a criterion for direct controller reduction is given?

The paper is organized as follows. Section 2 will spec- 


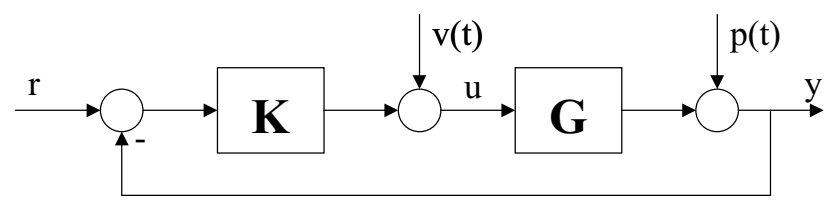

Figure 1: The closed-loop system

ify the notations. Section 3 will survey the various configurations for closed loop plant model identification and their properties. Section 4 will explore the various configurations for direct estimation in closed loop of reduced order controllers and their properties. Section 5 will give the basic algorithms and will indicate how they have to be modified in order to cover all the situations. Section 6 will discuss the interaction between closed loop identification and direct controller reduction.

\section{Notations}

Consider the system shown in Fig. 1 where the plant model is given by:

$$
G\left(z^{-1}\right)=\frac{z^{-d} B\left(z^{-1}\right)}{A\left(z^{-1}\right)}
$$

where:

$$
\begin{aligned}
A\left(z^{-1}\right) & =1+a_{1} z^{-1}+\cdots+a_{n_{A}} z^{-n_{A}} \\
& =1+z^{-1} A^{*}\left(z^{-1}\right) \\
B\left(z^{-1}\right) & =b_{1} z^{-1}+\cdots+b_{n_{B}} z^{-n_{B}} \\
& =z^{-1} B^{*}\left(z^{-1}\right)
\end{aligned}
$$

and the nominal controller by:

$$
K\left(z^{-1}\right)=\frac{R\left(z^{-1}\right)}{S\left(z^{-1}\right)}
$$

One defines the following sensitivity functions:

$$
\begin{aligned}
& \text { - } S_{y p}\left(z^{-1}\right)=\frac{1}{1+K G}=\frac{A\left(z^{-1}\right) S\left(z^{-1}\right)}{P\left(z^{-1}\right)} ; \\
& \text { - } S_{u p}\left(z^{-1}\right)=\frac{-K}{1+K G}=\frac{-A\left(z^{-1}\right) R\left(z^{-1}\right)}{P\left(z^{-1}\right)} ; \\
& \text { - } S_{y v}\left(z^{-1}\right)=\frac{G}{1+K G}=\frac{z^{-d} B\left(z^{-1}\right) S\left(z^{-1}\right)}{P\left(z^{-1}\right)} ; \\
& \text { - } S_{y r}\left(z^{-1}\right)=\frac{K G}{1+K G}=\frac{z^{-d} B\left(z^{-1}\right) R\left(z^{-1}\right)}{P\left(z^{-1}\right)} .
\end{aligned}
$$

where

$$
P\left(z^{-1}\right)=A\left(z^{-1}\right) S\left(z^{-1}\right)+z^{-d} B\left(z^{-1}\right) R\left(z^{-1}\right)
$$

The system of Fig.1 will be denoted the "true closed loop system". Throughout the paper we will consider feedback systems which will use either an estimation of $G$ (denoted $\hat{G}$ ) or a reduced order estimation of $K$ (denoted $\hat{K}$ ). The corresponding sensitivity functions will be denoted as follows:
- $S_{x y}$ - Sensitivity function of the true closed loop system $(K, G)$.

- $\hat{S}_{x y}$ - Sensitivity function of the nominal simulated closed loop system (nominal controller $K$ + estimated plant model $\hat{G}$ ).

- $\hat{\hat{S}}_{x y}$ - Sensitivity function of the simulated closed loop system using a reduced order controller (reduced controller $\hat{K}+$ estimated plant model $\hat{G}$ ).

Similar notations are used for $P\left(z^{-1}\right): \hat{P}\left(z^{-1}\right)$ when using $K$ and $\hat{G}, \hat{P}\left(z^{-1}\right)$ when using $\hat{K}$ and $\hat{G}$.

\section{Closed loop identification schemes}

Figures 2a, 2b and 2c summarize the various configurations belonging to the closed loop identification algorithms.

We will indicate next the properties of each configuration.

a) Closed loop output error with external excitation added to the reference signal: The identification criterion in this case is:

$$
\hat{\theta}^{*}=\arg \min _{\theta}\left\|S_{y r}-\hat{S}_{y r}\right\|_{2}=\arg \min _{\theta}\left\|S_{y p}-\hat{S}_{y p}\right\|_{2}
$$

and the asymptotic frequency distribution of the bias when the estimated plant model does not belong to the model set is given by $[3,4]$ :

$$
\begin{array}{r}
\hat{\theta}^{*}=\arg \min _{\theta} \int_{-\pi}^{\pi}\left|S_{y p}\right|^{2}\left[|G-\hat{G}|^{2}\left|\hat{S}_{u p}\right|^{2} \phi_{r}(\omega)\right. \\
\left.+\phi_{p}(\omega)\right] d \omega \\
=\arg \min _{\theta} \int_{-\pi}^{\pi}\left[\left|S_{y r}-\hat{S}_{y r}\right|^{2} \phi_{r}(\omega)\right. \\
\left.+\left|S_{y p}\right|^{2} \phi_{p}(\omega)\right] d \omega
\end{array}
$$

b) Closed loop output error with external excitation added to the plant input: The identification criterion in this case is:

$$
\hat{\theta}^{*}=\arg \min _{\theta}\left\|S_{y v}-\hat{S}_{y v}\right\|_{2}
$$

and the asymptotic distribution of the bias is given by:

$$
\begin{array}{r}
\hat{\theta}^{*}=\arg \min _{\theta} \int_{-\pi}^{\pi}\left|S_{y p}\right|^{2}\left[|G-\hat{G}|^{2}\left|\hat{S}_{y p}\right|^{2} \phi_{r}(\omega)\right. \\
\left.+\phi_{p}(\omega)\right] d \omega \\
=\arg \min _{\theta} \int_{-\pi}^{\pi}\left[\left|S_{y v}-\hat{S}_{y v}\right|^{2} \phi_{r}(\omega)\right. \\
\left.+\left|S_{y p}\right|^{2} \phi_{p}(\omega)\right] d \omega
\end{array}
$$

c) Closed loop input error with external excitation added to the reference: The identification criterion in this case is:

$$
\hat{\theta}^{*}=\arg \min _{\theta}\left\|S_{u p}-\hat{S}_{u p}\right\|_{2}
$$




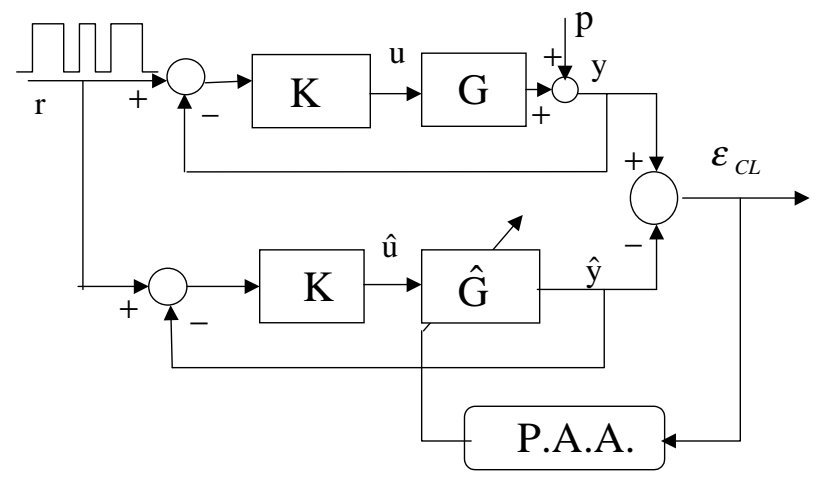

Fig. 2a Closed loop output error (CLOE) (external

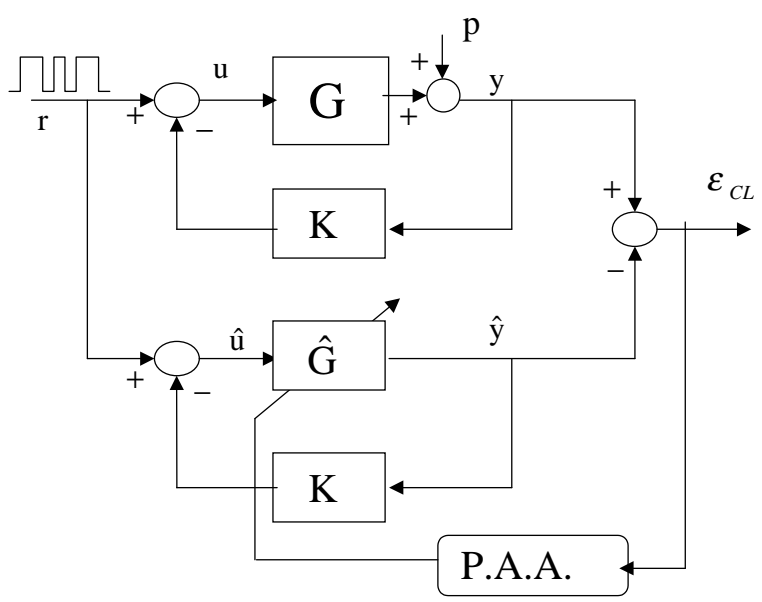

Fig. 2b Closed loop output error (CLOE) (external

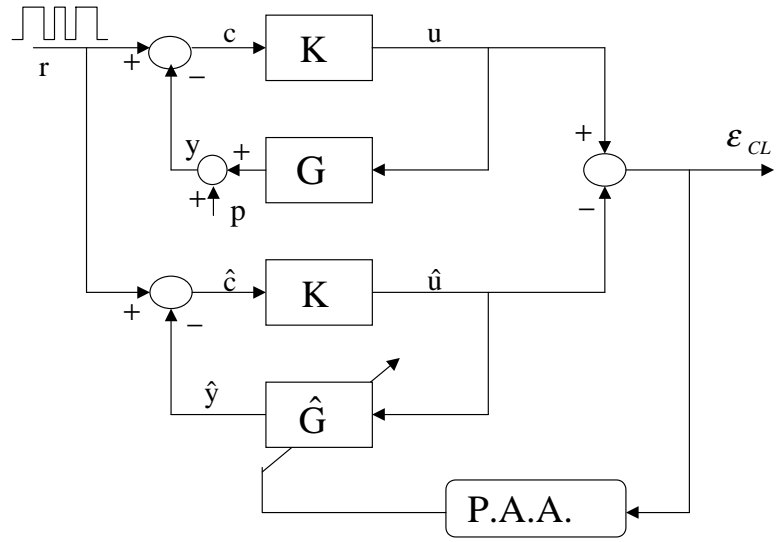

Fig. 2c Closed loop input error (CLIE) (external excitation added to the reference) and the asymptotic distribution of the bias is given by:

$$
\begin{aligned}
\hat{\theta}^{*} & =\arg \min _{\theta} \int_{-\pi}^{\pi}\left[\left|S_{u p}-\hat{S}_{u p}\right|^{2} \phi_{r}(\omega)\right. \\
& \left.+\left|S_{u p}\right|^{2} \phi_{p}(\omega)\right] d \omega
\end{aligned}
$$

Note that one can consider a fourth configuration corresponding to a closed loop input error with external excitation added to the plant input. However this configuration corresponds to the one of Fig.2a where in the upper part (the true system) the place of $K$ and $\mathrm{G}$ is reverted. This however will not change the identification criterion and will not affect the asymptotic frequency distribution of the bias.

In short for the configuration of Fig. $2 \mathrm{a}, 2 \mathrm{~b}$ and $2 \mathrm{c}$, if $r(t)$ is a discrete time white noise (for example a PRBS which is a good approximation) the algorithm will search for the best $\hat{G}$ which will minimize the 2norm between the various sensitivity functions. In addition the differences between $\hat{G}$ and $\mathrm{G}$ will be minimized in the frequency regions where the sensitivity functions have large values.

The practical consequence of these properties is that the choice of one scheme or another is related to the main performance objective of the controller design. For tracking and output disturbance rejection it is preferable to use the scheme of Fig. 2a. For minimization of the effect of the output disturbance on the controller input it is preferable to use the scheme of Fig. 2c. For output rejection of a disturbance entered at the input of the plant it is preferable to use the scheme of Fig. $2 \mathrm{~b}$.

\section{Direct estimation in closed loop of reduced order controllers}

We will consider that a model of the plant is available (denoted by $\hat{G}$ ). This model can be the plant model used for design or an identified model (in open loop or in closed loop) which passed the validation tests.

We will assume of course that the nominal controller of orders $n_{R}$ and $n_{S}$ is known (denoted by $K$ ).

In fact in the context of this paper we will assume that the model was identified in closed loop with the nominal controller since almost always the closed loop identification with the nominal controller will give a model which will be better than the open loop identified in the sense of the identification criteria (6), (8) and (10).

Figures $3 \mathrm{a}, 3 \mathrm{~b}$ and $3 \mathrm{c}$ summarize the various configuration for direct estimation in closed loop of reduced order controllers. We will indicate next the properties of each configuration.

a) Closed loop input matching with external excitation added to the plant input: The controller reduction cri- 


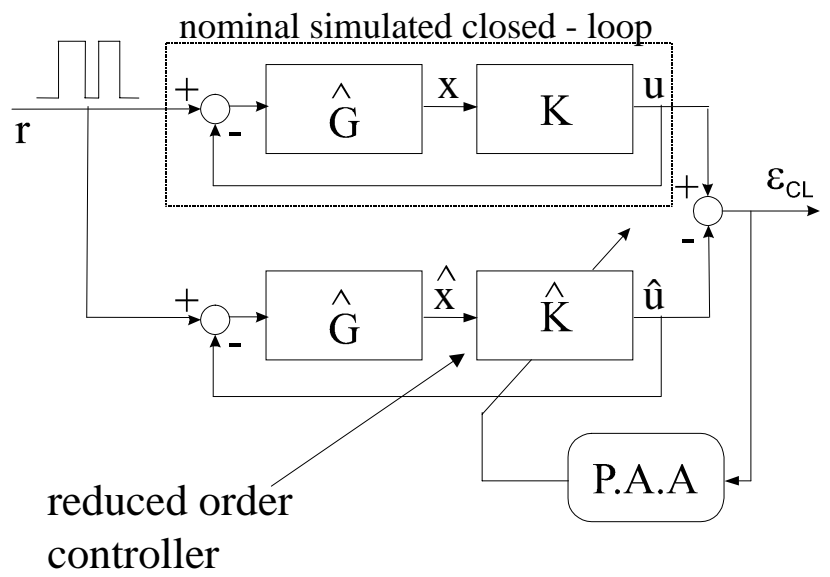

Fig. 3a Closed loop input matching (CLIM) (external excitation added to the plant input)

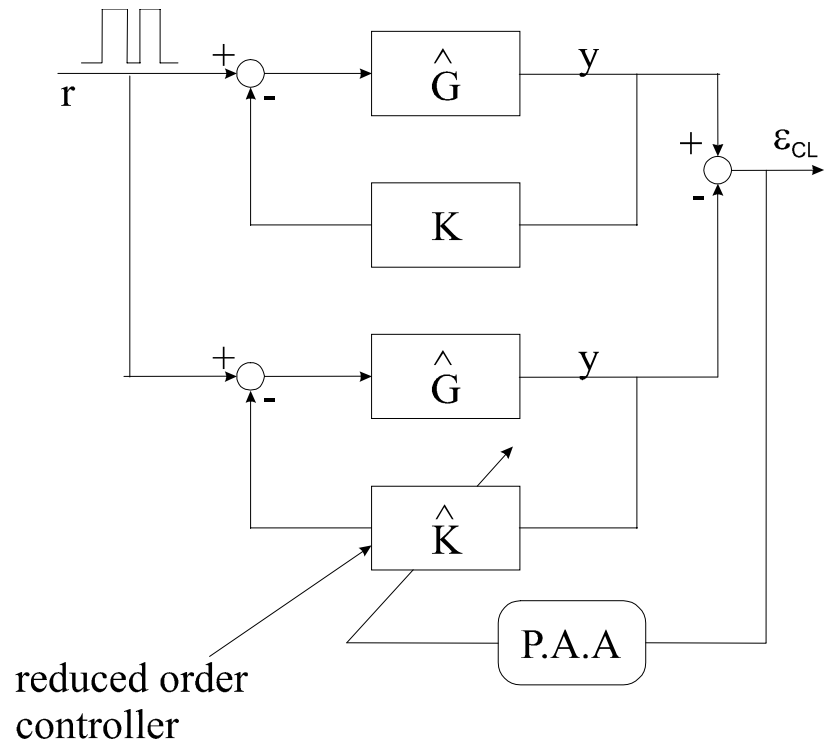

Fig. 3b Closed loop output matching (CLOM) (external excitation added to the plant input)

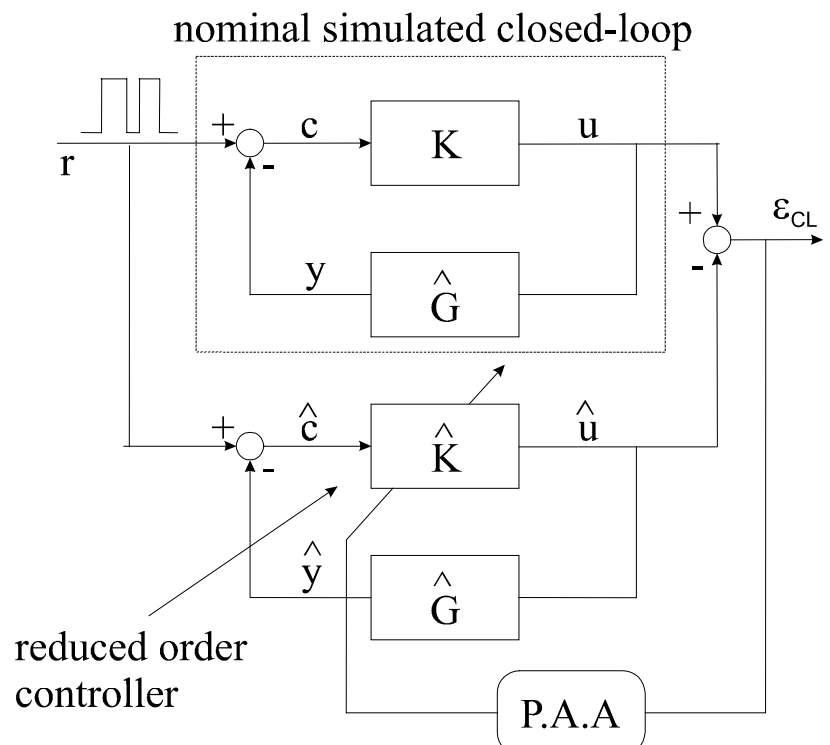

Fig. 3c Closed loop input matching (CLIM) (external excitation added to the reference) terion in this case is:

$$
\hat{\theta}_{c}^{*}=\arg \min _{\theta_{c}}\left\|\hat{S}_{y r}-\hat{\hat{S}}_{y r}\right\|_{2}=\arg \min _{\theta_{c}}\left\|\hat{S}_{y p}-\hat{\hat{S}}_{y p}\right\|_{2}
$$

and the asymptotic frequency distribution of the bias is given by $[3,5]$ :

$$
\begin{aligned}
\hat{\theta}_{c}^{*} & =\arg \min _{\theta_{c}} \int_{-\pi}^{\pi}\left|\hat{S}_{y p}\right|^{2}|K-\hat{K}|^{2}\left|\hat{S}_{y v}\right| \phi_{r}(\omega) d \omega \\
& =\arg \min _{\theta_{c}} \int_{-\pi}^{\pi}\left|\hat{S}_{y r}-\hat{\hat{S}}_{y r}\right|^{2} \phi_{r}(\omega) d \omega
\end{aligned}
$$

b) Closed loop output matching with external excitation added to the plant input: The controller reduction criterion in this case is:

$$
\hat{\theta}_{c}^{*}=\arg \min _{\theta_{c}}\left\|\hat{S}_{y v}-\hat{\hat{S}}_{y v}\right\|_{2}
$$

and the asymptotic frequency distribution of the bias is given by:

$$
\hat{\theta}_{c}^{*}=\arg \min _{\theta_{c}} \int_{-\pi}^{\pi}\left|\hat{S}_{y r}-\hat{\hat{S}}_{y r}\right|^{2} \phi_{r}(\omega) d \omega
$$

c) Closed loop input matching with external excitation added to the reference: The controller reduction criterion in this case is:

$$
\hat{\theta}_{c}^{*}=\arg \min _{\theta_{c}}\left\|\hat{S}_{u p}-\hat{\hat{S}}_{u p}\right\|_{2}
$$

and the asymptotic frequency distribution of the bias is given by:

$$
\begin{aligned}
\hat{\theta}_{c}^{*} & =\arg \min _{\theta_{c}} \int_{-\pi}^{\pi}\left|\hat{S}_{y p}\right|^{2}|K-\hat{K}|^{2}\left|\hat{\hat{S}}_{y p}\right| \phi_{r}(\omega) d \omega \\
& =\arg \min _{\theta_{c}} \int_{-\pi}^{\pi}\left|\hat{S}_{u p}-\hat{\hat{S}}_{u p}\right|^{2} \phi_{r}(\omega) d \omega
\end{aligned}
$$

Note that one can consider a fourth configuration corresponding to a closed loop output matching scheme with external excitation added to the reference. However this configuration corresponds to the one of Fig 3 a where in the upper (the nominal simulated system) the place of $K$ and $\hat{G}$ is reverted.

When $r(t)$ is a discrete white noise (for example a PBRS) the algorithms will search for the best controller $\hat{K}$ which will minimize the 2 -norm reduction criterion.

Like in closed loop identification the differences between $K$ and $\hat{K}$ will be small at the frequencies where the sensitivity functions have large values.

The above schemes can be used also with real data $[3,5]$. In this case it can be shown that the noise will not affect the minimization procedure. 


\section{Closed loop output error identification algorithms (CLOE)}

The two basic schemes are shown in Fig. 2a and Fig. $2 \mathrm{~b}$.

The output of the plant is given by:

$$
\begin{aligned}
& y(t+1)=-A^{*} y(t)+B^{*} u(t-d)=\theta^{T} \psi(t) \\
& \psi(t)=\left[-y(t), \ldots,-y\left(t-n_{A}+1\right), u(t-d)\right. \\
&\left.\ldots, u\left(t-d-n_{B}+1\right)\right] \\
& \theta=\left[a_{1}, \ldots, a_{n_{A}}, b_{1}, \ldots, b_{n_{B}}\right] \\
& u(t)=-\frac{R}{S} y(t)+r_{u}(t)
\end{aligned}
$$

where $r_{u}(t)=r(t)$ in the scheme of Fig.2b and $r_{u}(t)=$ $\frac{R}{S} r(t)$ in the scheme of Fig. $2 \mathrm{a}$.

The output of the closed loop adjustable predictor is given by:

a priori:

$$
\begin{aligned}
\hat{y}^{0}(t+1) & =-\hat{A}^{*}\left(t, q^{-1}\right) \hat{y}(t)+\hat{B}^{*}\left(t, q^{-1}\right) \hat{u}(t-d) \\
& =\hat{\theta}^{T}(t) \phi(t) \\
\hat{u}(t) & =-\frac{R\left(q^{-1}\right)}{S\left(q^{-1}\right)} \hat{y}(t)+r_{u}(t)
\end{aligned}
$$

a posteriori:

$$
\hat{y}(t+1)=\hat{\theta}^{T}(t+1) \phi(t)
$$

where

$$
\begin{aligned}
& \hat{\theta}^{T}(t)= {\left[\hat{a}_{1}, \ldots, \hat{a}_{n_{\hat{A}}}, \hat{b}_{1}, \ldots, \hat{b}_{n_{\hat{B}}}\right] } \\
& \phi(t)=\left[-\hat{y}(t), \ldots,-\hat{y}\left(t-n_{\hat{A}}+1\right), \hat{u}(t-d),\right. \\
&\left.\ldots, \hat{u}\left(t-d-n_{\hat{B}}+1\right)\right]
\end{aligned}
$$

The closed loop output error is defined as:

a priori: $\quad \varepsilon_{C L}^{0}(t+1)=y(t+1)-\hat{y}^{0}(t+1)$

a posteriori: $\quad \varepsilon_{C L}(t+1)=y(t+1)-\hat{y}(t+1)$

and the parameter adaptation algorithm (P.A.A.) will be given by:

$$
\begin{aligned}
& \hat{\theta}(t+1)=\hat{\theta}(t)+F(t) \Phi(t) \varepsilon_{C L}(t+1) \\
& F^{-1}(t+1)=\lambda_{1}(t) F^{-1}(t)+\lambda_{2}(t) \Phi(t) \Phi^{T}(t)(30) \\
& \quad 0<\lambda_{1}(t) \leq 1 ; 0 \leq \lambda_{2}(t)<2 ; \quad F(0)>0 \\
& \varepsilon_{C L}(t+1)=\frac{\varepsilon_{C L}^{0}(t+1)}{1+\Phi^{T}(t) F(t) \Phi(t)}
\end{aligned}
$$

Specific algorithms are obtained by an appropriate choice of the observation vector $\Phi(t)$ as follows:

$$
\begin{array}{ll}
\text { CLOE } & \Phi(t)=\phi(t) \\
\text { F-CLOE } & \Phi(t)=\frac{S\left(q^{-1}\right)}{\hat{P}\left(q^{-1}\right)} \phi(t) \\
\text { AF-CLOE } & \Phi(t)=\frac{S\left(q^{-1}\right)}{\hat{P}\left(t, q^{-1}\right)} \phi(t)
\end{array}
$$

where:

$$
\begin{aligned}
\hat{P}\left(q^{-1}\right) & =\hat{A}\left(q^{-1}\right) S\left(q^{-1}\right)+q^{-d} \hat{B}\left(q^{-1}\right) R\left(q^{-1}\right) \\
\hat{P}\left(t, q^{-1}\right) & =\hat{A}\left(t, q^{-1}\right) S\left(q^{-1}\right)+q^{-d} \hat{B}\left(t, q^{-1}\right) R\left(q^{-1}\right)
\end{aligned}
$$

$\hat{A}\left(q^{-1}\right)$ and $\hat{B}\left(q^{-1}\right)$ correspond to an a priori estimation of $\hat{G}$, while $\hat{A}\left(t, q^{-1}\right)$ and $\hat{B}\left(t, q^{-1}\right)$ correspond to the current estimates of $\hat{G}$.

When $n_{\hat{A}}=n_{A}, n_{\hat{B}}=n_{B}$, then the closed loop output error goes to zero (in a deterministic environment) and unbiased estimates are obtained in a stochastic environment (when $p(t)$ is independent with respect to $r_{u}(t)$ and of finite power) if:

$$
H^{\prime}\left(z^{-1}\right)=H\left(z^{-1}\right)-\frac{\lambda}{2} ; \max _{t} \lambda_{2}(t) \leq \lambda<2
$$

is a strictly positive real transfer function, where:

$$
H\left(z^{-1}\right)=\left\{\begin{array}{l}
S / P \text { for } C L O E \\
\hat{P} / P \text { for } F-C L O E \\
1 \text { for } A F-C L O E
\end{array}\right.
$$

(in the last case this is a local result) $[4,6]$.

\section{Algorithms for direct estimation in closed loop of reduced order controllers}

These algorithms are obtained from the closed loop identification algorithms by making dual type modifications in the scheme of figures $2 \mathrm{a}$ and $2 \mathrm{~b}$ summarized in Table 1. The resulting schemes are shown in Fig.3a and Fig.3b.

\begin{tabular}{|c|c|c|}
\hline $\begin{array}{c}\text { Plant model } \\
\text { identification } \\
\text { in closed loop } \\
\text { Fig.2b, Fig.2a }\end{array}$ & & $\begin{array}{c}\text { Identification of } \\
\text { reduced order controller } \\
\text { in closed loop } \\
\text { Fig.3c, Fig.3a }\end{array}$ \\
\hline \hline $\begin{array}{c}\text { controller } \\
(K)\end{array}$ & $\longrightarrow$ & $\begin{array}{c}\text { available plant } \\
\text { model }(\hat{G})\end{array}$ \\
\hline $\begin{array}{c}\text { true plant } \\
\text { model }(G)\end{array}$ & $\longrightarrow$ & $\begin{array}{c}\text { nominal } \\
\text { controller }(K)\end{array}$ \\
\hline $\begin{array}{c}\text { estimated } \\
\text { plant model } \\
(\hat{G})\end{array}$ & $\longrightarrow$ & $\begin{array}{c}\text { estimated (reduced } \\
\text { order }) \text { controller } \\
(\hat{K})\end{array}$ \\
\hline$y, \hat{y}$ & $\longrightarrow$ & $u, \hat{u}$ \\
\hline$u, \hat{u}$ & $\longrightarrow$ & $x, \hat{x}$ \\
\hline \multicolumn{2}{|c|}{}
\end{tabular}

Table 1: Duality between plant model identification in closed loop and direct estimation of reduced order controller in closed loop

The signal $x(t)$ is defined as:

$$
x(t)=r(t)-u(t)
$$

in Fig. $3 \mathrm{c}$ and

$$
x(t)=\hat{G}[r(t)-u(t)]
$$

in Fig. 3a. 
As it will be shown, the scheme of Fig. 3c corresponds to closed loop input matching objective (CLIM algorithm) and the scheme of Fig. 3a corresponds to closed loop output matching objective (CLOM algorithm) for controller reduction.

With the above changes one has

a priori:

$$
\begin{aligned}
\hat{u}^{0}(t+1) & =-\hat{S}^{*}\left(t, q^{-1}\right) \hat{u}(t)+\hat{R}\left(t, q^{-1}\right) \hat{x}(t) \\
& =\hat{\theta}_{c}^{T}(t) \phi_{c}(t)
\end{aligned}
$$

a posteriori: $\quad \hat{u}(t+1)=\hat{\theta}_{c}^{T}(t+1) \phi_{c}(t)$

where

$$
\begin{aligned}
\hat{x}(t+1)= & -\hat{A}^{*}\left(q^{-1}\right) \hat{x}(t)-\hat{B}^{*}\left(q^{-1}\right) \hat{u}(t-d) \\
& +A\left(q^{-1}\right) r(t)
\end{aligned}
$$

for the scheme of Fig. 3c,

$$
\begin{aligned}
\hat{x}(t+1)= & -\hat{A}^{*}\left(q^{-1}\right) \hat{x}(t)-\hat{B}^{*}\left(q^{-1}\right) \hat{u}(t-d) \\
& +\hat{B}^{*} r(t-d)
\end{aligned}
$$

for the scheme of Fig. 3a, and

$$
\begin{gathered}
\hat{\theta}_{c}^{T}(t)=\left[\hat{s}_{1}(t), \ldots, \hat{s}_{n_{\hat{S}}}(t), \hat{r}_{0}(t), \ldots, \hat{r}_{n_{\hat{R}}}(t)\right] \\
\phi_{c}(t)=\left[-\hat{u}(t), \ldots,-\hat{u}\left(t-n_{\hat{S}}+1\right), \hat{x}(t+1),\right. \\
\left.\ldots, \hat{x}\left(t-n_{\hat{R}}+1\right)\right]
\end{gathered}
$$

The closed loop input error will be given by:

$$
\begin{array}{ll}
\text { a priori: } & \varepsilon_{C L}^{0}(t+1)=u(t+1)-\hat{u}^{0}(t+1) \\
\text { a posteriori: } & \varepsilon_{C L}(t+1)=u(t+1)-\hat{u}(t+1)
\end{array}
$$

and the same P.A.A. algorithm described in the Eqs. (29) through (31) can be used and the corresponding specific algorithms will be:

$$
\begin{array}{ll}
\text { CLIM / CLOM } & \Phi(t)=\phi_{c}(t) \\
\text { F-CLIM / F-CLOM } & \Phi(t)=\frac{\hat{A}\left(q^{-1}\right)}{\hat{P}\left(q^{-1}\right)} \phi_{c}(t)
\end{array}
$$

where:

$$
\hat{P}\left(q^{-1}\right)=\hat{A}\left(q^{-1}\right) S\left(q^{-1}\right)+q^{-d-1} \hat{B}\left(q^{-1}\right) R\left(q^{-1}\right)
$$

is a known quantity and therefore there is no need to estimate this polynomial in line.

The corresponding transfer functions involved in the passivity conditions for stability becomes:

$$
H\left(z^{-1}\right)= \begin{cases}\frac{\hat{A}\left(q^{-1}\right)}{\hat{P}\left(q^{-1}\right)} & \text { for } \quad \text { CLIM/CLOM } \\ 1 \text { for } & \mathrm{F}-\mathrm{CLIM} / \mathrm{F}-\mathrm{CLOM}\end{cases}
$$

(since the exact polynomial of the nominal simulated closed loop is known).

\section{Choice of the appropriate identification algorithm for direct controller reduction}

The interesting problem to address is: What closed loop plant model identification should be used when a

\begin{tabular}{|c|c|c|}
\hline $\begin{array}{l}\text { Controller } \\
\text { reduction } \\
\text { criterion }\end{array}$ & $\begin{array}{l}\text { Controller } \\
\text { reduction } \\
\text { scheme }\end{array}$ & $\begin{array}{c}\text { Closed loop } \\
\text { identification } \\
\text { scheme }\end{array}$ \\
\hline \multirow{4}{*}{$\begin{array}{c}\min \left\|\hat{S}_{y p}-\hat{S}_{y p}\right\| \\
\text { or } \\
\min \left\|\hat{S}_{y r}-\hat{S}_{y r}\right\|\end{array}$} & CLIM with & CLOE with \\
\hline & external & external \\
\hline & excitation & excitation \\
\hline & $\begin{array}{l}\text { added to the } \\
\text { plant input }\end{array}$ & $\begin{array}{l}\text { added to the } \\
\text { reference }\end{array}$ \\
\hline \multirow{3}{*}{$\min \left\|\hat{S}_{y v}-\hat{\hat{S}}_{y v}\right\|$} & $\begin{array}{c}\text { CLOM with } \\
\text { external }\end{array}$ & $\begin{array}{c}\text { CLOE with } \\
\text { external }\end{array}$ \\
\hline & excitation & excitation \\
\hline & $\begin{array}{l}\text { added to the } \\
\text { plant input }\end{array}$ & $\begin{array}{l}\text { added to the } \\
\text { plant input }\end{array}$ \\
\hline \multirow{3}{*}{$\min \left\|\hat{S}_{u p}-\hat{\hat{S}}_{u p}\right\|$} & $\begin{array}{l}\text { CLIM with } \\
\text { external }\end{array}$ & $\begin{array}{c}\text { CLIE with } \\
\text { external }\end{array}$ \\
\hline & excitation & excitation \\
\hline & $\begin{array}{c}\text { added to the } \\
\text { reference }\end{array}$ & $\begin{array}{l}\text { added to the } \\
\text { reference }\end{array}$ \\
\hline
\end{tabular}
criterion for direct controller reduction is given?

To answer this question we will make reference to the iterative identification in closed loop and controller redesign methodology [2]. The basic rule for improving performance is to use the same criterion for identification in closed loop and controller re-design. In our case controller re-design corresponds to the reduction of the nominal controller.

Therefore examining the identification criterion for the various closed loop identification schemes and the controller reduction objectives they watch each other as indicated in Table 2.

Table 2: Coherent controller reduction and identification in closed loop

\section{Conclusions}

A unified approach to closed-loop plant identification and direct controller reduction by closed-loop identification has been proposed. The main idea is to consider the control objective in all of the model based control design steps (i.e. in model identification step, in high order controller design and in the controller reduction step). The control criterion can be matched with the identification criterion by plant model identification in closed-loop with the appropriate choice of the criterion (output error or input error) as well as the place where the excitation signal is added (to the reference signal or to the control input). The control objective can also be considered in the controller reduction step by direct identification of a reduced order controller in closedloop. In this step the choice of the criterion and the 
excitation point play an important role. It has been shown that the algorithms for controller reduction can be derived by duality from the algorithms for plant model identification in closed-loop.

\section{References}

[1] U. Forssell and L. Ljung. Closed loop identification revisited. Automatica, 35(7):1215-1242, 1999.

[2] M. Gevers. Towards a joint design of identification and control, volume Essays on Control: Perspectives in the Theory and its applications. H.L. Trentelman, J.C. Willems Editors, Birkhauser, pp. 111-151 1993.

[3] A. Karimi and I.D. Landau. Controller order reduction by direct closed loop identification (output matching). IFAC Symp. ROCOND 2000, Prague June 2000.

[4] I.D. Landau and A. Karimi. Recursive algorithms for identification in closed loop - a unified approach and evaluation. Automatica, 33(8):1499$1523,1997$.

[5] I.D. Landau and A. Karimi. Direct closed loop identification of reduced order controllers (input matching). IFAC Symp. SYSID 2000, S. Barbara June 2000.

[6] I.D. Landau, R. Lozano, and M. M'Saad. Adaptive Control. Springer, London, 1997. 